\title{
CNS Penetration of Cyclophosphamide and Metabolites in Mice Bearing Group 3 Medulloblastoma and Non-Tumor Bearing Mice
}

\author{
Olivia Campagne ${ }^{1}$, Abigail Davis ${ }^{1}$, Bo Zhong ${ }^{1}$, Sreenath Nair ${ }^{1}$, Victoria Haberman ${ }^{1}$, Yogesh T. Patel ${ }^{2}$, Laura Janke ${ }^{3}$, Martine \\ F. Roussel ${ }^{4}$, Clinton F. Stewart ${ }^{1}$ \\ 1. Pharmaceutical Sciences Department, St. Jude Children's Research Hospital, Memphis TN. ${ }^{2}$ Pharmaceutical Sciences \\ Department, St. Jude Children's Research Hospital, Memphis TN. (currently at Cognigen Corp., Buffalo, NY). \\ 3. Department of Veterinary Pathology Core, St. Jude Children's Research Hospital, Memphis TN. ${ }^{4}$ Department of Tumor \\ Cell Biology, St. Jude Children's Research Hospital, Memphis TN
}

Received, July 1, 2019; Revised, September 18, 2019; Accepted, September 29, 2019; Published, December 4, 2019.

\begin{abstract}
PURPOSE: Cyclophosphamide is widely used to treat children with medulloblastoma; however, little is known about its brain penetration. We performed cerebral microdialysis to characterize the brain penetration of cyclophosphamide (130 mg/kg, IP) and its metabolites [4-hydroxy-cyclophosphamide (4OH-CTX) and carboxyethylphosphoramide mustard (CEPM)] in non-tumor bearing mice and mice bearing orthotopic Group 3 medulloblastoma. METHODS: A plasma pharmacokinetic study was performed in non-tumor-bearing CD1nude mice, and four cerebral microdialysis studies were performed in non-tumor-bearing (M1 and M3) and tumorbearing mice (M2 and M4). Plasma samples were collected up to 6-hours post-dose, and extracellular fluid (ECF) samples were collected over 60 -minute intervals for 24-hours post-dose. To stabilize and quantify $4 \mathrm{OH}-\mathrm{CTX}$, a derivatizing solution was added in blood after collection, and either directly in the microdialysis perfusate (M1 and M2) or in ECF collection tubes (M3 and M4). Plasma/ECF cyclophosphamide and CEPM, and 4OH-CTX concentrations were separately measured using different LC-MS/MS methods. RESULTS: All plasma/ECF concentrations were described using a population-based pharmacokinetic model. Plasma exposures of cyclophosphamide, 4OH-CTX, and CEPM were similar across studies (mean AUC=112.6, 45.6, and 80.8 $\mu \mathrm{mol} \cdot \mathrm{hr} / \mathrm{L})$. Hemorrhage was observed in brain tissue when the derivatizing solution was in perfusate compared with none when in collection tubes, which suggested potential sample contamination in studies M1 and M2. Model-derived unbound ECF to plasma partition coefficients $\left(\mathrm{K}_{\mathrm{p}, \mathrm{uu}}\right)$ were calculated to reflect CNS penetration of the compounds. Lower cyclophosphamide $\mathrm{K}_{\mathrm{p} \text {,u }}$ was obtained in tumor-bearing mice versus non-tumor bearing mice (mean 0.15 versus 0.22 , $\mathrm{p}=0.019$ ). No differences in $\mathrm{K}_{\mathrm{p} \text {,uu }}$ were observed between these groups for $4 \mathrm{OH}$ CTX and CEPM (overall mean 0.10 and 0.07). CONCLUSIONS: Future studies will explore potential mechanisms at the brain-tumor barrier to explain lower cyclophosphamide brain penetration in tumor-bearing mice. These results will be used to further investigate exposure-response relationships in medulloblastoma xenograft models.
\end{abstract}

\section{INTRODUCTION}

Medulloblastoma is the most common malignant brain cancer in children (1). These brain tumors are highly heterogeneous, and divided molecularly into four major subgroups termed Wingless, Sonic hedgehog, Group 3, and Group 4, with distinctive outcome, transcriptional and epigenetic signatures (2-4). Contemporary therapy for patients with medulloblastoma includes surgery, radiation therapy to the whole craniospinal axis, and high-dose cytotoxic chemotherapy. This approach has led over the years to a cure rate exceeding $70 \%$ for average risk tumors, but only $30 \%$ for Group 3 medulloblastoma (G3MB) (5).
Cyclophosphamide has demonstrated efficacy against medulloblastoma preclinically and clinically since the early $1980 \mathrm{~s}$, and is considered as a standard of care along with vincristine and cisplatin (6-8). As such, cyclophosphamide is frequently used in efficacy studies in mouse models of G3MB to investigate the effects of potential new combination chemotherapies (9-11).

Corresponding Author: Clinton F. Stewart, Pharm.D., Department of Pharmaceutical Sciences, St. Jude Children's Research Hospital, 262 Danny Thomas Place, Memphis, TN 38105-2794, USA. Telephone: (901) 595-3665; FAX: (901) 525-6869 E-mail: clinton.stewart@stjude.org 
Although its efficacy in treating medulloblastoma suggests an adequate central nervous system (CNS) penetration of the compound, very little has been published in this setting, and only in healthy rats and monkeys (12-14). No investigation has yet been done to characterize the penetration of cyclophosphamide across the blood-brain-barrier or the blood-tumor barrier in mice. This information would be valuable to aid in characterizing the exposure-response relationships in the mouse tumor models, and explore new combination treatments.

The inactive parent drug cyclophosphamide undergoes complex hepatic metabolism to form numerous metabolites through which it exerts cytotoxic activity (15). Thus, it is important to study the parent drug as well as its active metabolite(s) to better understand the antitumor efficacy. In humans, cyclophosphamide is predominantly metabolized to 4-hydroxy-cyclophosphamide (4OH-CTX) by multiple cytochrome P450 enzymes, which can then diffuse into cells and be converted into phosphoramide mustard, the ultimate alkylating metabolite. 4OH-CTX is commonly measured as a surrogate to investigate the drug cytotoxic activity, although its bioanalysis requires a specific procedure $(16,17)$. $4 \mathrm{OH}-\mathrm{CTX}$ is very reactive and unstable in biologic fluids and needs to be immediately stabilized with a phenylhydrazine derivatizing solution after sample collection. This metabolite can also be converted by aldehyde dehydrogenase to inactive carboxyethylphosphoramide mustard (CEPM) (15).

A preclinical approach using cerebral microdialysis along with pharmacokinetic modeling and simulation was previously developed and applied to characterize the brain penetration of drugs in tumor subgroup-specific models of CNS tumors (18). Cerebral microdialysis allows the collection of brain extracellular fluid (ECF) samples and the measurement of unbound drug concentrations in ECF over a set amount of time. As mentioned previously, measuring 4OH-CTX ECF sample would require the addition of the derivatizing solution during the cerebral microdialysis process. In this setting, the derivatizing solution could be added either in the microdialysis probe perfusate or after sample collection in the tubes.

In the current study, we applied a preclinical approach in which plasma and ECF disposition, and plasma protein binding of cyclophosphamide and its metabolites, were characterized in both non-tumor- bearing mice and mice orthotopically implanted with G3MB. The potential impact of the different methods to collect 4OH-CTX ECF samples (i.e., derivatizing solution in perfusate versus collection tube) on its pharmacokinetic profile was evaluated. Last, the ECF to plasma partition coefficient $\left(\mathrm{K}_{\mathrm{p}, \mathrm{uu}}\right)$ reflecting the CNS penetration was estimated for each compound using pharmacokinetic modeling in both non-tumor and tumor-bearing mice.

\section{METHODS}

\section{Chemicals and drugs}

Cyclophosphamide monohydrate was purchased from Millipore Sigma (St. Louis, MO), cyclophosphamide- $d_{4}$ from Medical Isotopes (Pelham, NH), CEPM, and 4OH-CTX from Niomech (Bielefeld, Germany). Carboxyethylphosphoramide- $d_{8}$ was obtained as a custom synthesis from Chemical Biology and Therapeutics of St. Jude Children's Research Hospital. Formic acid (LC-MS/MS grade, 99.5\% purity), acetonitrile (HPLC grade), methanol (HPLC grade), sodium hydroxide (98.9 purity) and sodium thiosulfate pentahydrate $(99.8 \%$ purity) were purchased from Fisher Scientific (Waltham, MA). Ammonium acetate (for mass spectrometry, $\geq 99.0 \%$ ), phenylhydrazine hydrochloride $(\geq 99 \%$ purity), and sodium citrate tribasic dihydrate $(\geq$ 99.9\% purity) were purchased from Millipore Sigma (St. Louis, MO). CD-1 mouse plasma with sodium heparin as anticoagulant was purchased from BioreclamationIVT (Baltimore, MD) and lab grade Ringer's solution from Frey Scientific (Nashua, $\mathrm{NH})$. Water was prepared using Millipore Qadvantage water purification system (Temecula, CA). The derivatizing solution containing $277 \mathrm{mM}$ phenylhydrazine was prepared by dissolving phenyldrazine hydrochloride powder into $0.1 \mathrm{M}$ sodium citrate solution followed by adjusting the $\mathrm{pH}$ to 6 by addition of $10 \mathrm{M}$ sodium hydroxide solution. Cyclophosphamide for dosing was obtained from Baxter (Deerfield, IL). 0.9\% sodium chloride was purchased from APP Pharmaceuticals, LLC (Schaumburg, IL). Cyclophosphamide was formulated at a concentration of $13 \mathrm{mg} / \mathrm{mL}$ in $0.9 \%$ sodium chloride.

\section{Animals and tumor implantation}

CD1-nude mice were purchased from Charles River Laboratories (Willington, MA). $1 \times 10^{5}$ mouse G3MB 
purified tumor cells retrovirally transduced with luciferase were stereotactically implanted in the cortices of naive mice. Animals were kept under controlled environment where temperature, humidity, and $12 \mathrm{~h}$ day and night cycles were maintained artificially. All animal studies performed were approved by the St. Jude Children's Research Hospital Institutional Animal Care and Usage Committee (IACUC), and met the guidelines of the Association for Assessment and Accreditation of Laboratory Animal Care (AALAC).

\section{Plasma protein binding studies}

A plasma protein binding study of cyclophosphamide, 4OH-CTX, and CEPM was performed using a rapid equilibrium dialysis procedure to determine the unbound fraction $\left(\mathrm{F}_{\mathrm{U}}\right)$ of each analyte in mouse plasma. Each compound was added to CD1 mouse plasma to make final concentrations of 10 and $500 \mu \mathrm{M}$ for cyclophosphamide, 1 and $30 \mu \mathrm{M}$ for CEPM, and 0.5 and $15 \mu \mathrm{M}$ for $4 \mathrm{OH}-\mathrm{CTX} .1 \mathrm{~mL}$ of spiked plasma was added to Centrifree ${ }^{\circledR}$ ultrafiltration devices [molecular weight cut off (MQCO): $10 \mathrm{~K}$ Daltons; MilliporeSigma, Darmstadt, Germany]. The plasma samples were centrifuged for $30 \mathrm{~min}$ at $3600 \mathrm{rpm}$ at $37^{\circ} \mathrm{C}$. The filtrate was saved and stored at $-80^{\circ} \mathrm{C}$ until analysis. For each analyte, $\mathrm{F}_{\mathrm{U}}$ was calculated as ratio of unbound drug concentration in the filtrate to total drug concentration in the plasma sample.

\section{Plasma pharmacokinetic study}

A plasma pharmacokinetic study was performed in twelve female non-tumor-bearing CD1-nude mice dosed with $130 \mathrm{mg} / \mathrm{kg}$ cyclophosphamide via IP injection. A population-based study design was implemented where multiple samples were collected per mouse. Animals were divided into 4 groups (3 mice/group) with different plasma collection time points after injection: $5 \mathrm{~min}, 1.5$, and $4 \mathrm{~h}$ (cohort 1 ); 1 , and $6 \mathrm{~h}$ (cohort 2); 0.5 , and $4 \mathrm{~h}$ (cohort 3 ); and $0.25,2$, and $6 \mathrm{~h}$ (cohort 4$)$. The last blood sample (1 $\mathrm{mL}$ ) for each cohort was collected via cardiac stick, whereas all other samples $(75 \mu \mathrm{L})$ were collected via retro-orbital eye bleed.

Derivatizing solution $(277 \quad \mathrm{mM}$ phenylhydrazine) was immediately added to blood for $4 \mathrm{OH}-\mathrm{CTX}$ bioanalysis $(15 \mu \mathrm{L}$ of blood into 30 $\mu \mathrm{L}$ of derivatizing solution for blood samples collected via retro-orbital eye bleed, and $500 \mu \mathrm{L}$ of blood in $1 \mathrm{~mL}$ of derivatizing solution for blood samples collected via cardiac stick). The remainder of the blood was placed in a separate tube for both cyclophosphamide and CEPM bioanalysis. Both tubes were immediately spun to plasma, transferred into labeled $1.5 \mathrm{~mL}$ flip top tubes, and then stored at $-80^{\circ} \mathrm{C}$ until analysis.

\section{Cerebral microdialysis studies}

Four cerebral microdialysis studies were performed in non-tumor bearing mice (study M1 [n=5] and study M3 [n=9]) or mice that were orthotopically implanted with G3MB (study M2 [n=8] and study M4 [n=7]). In studies M1 and M3, mice were implanted with a cannula and enrolled in the microdialysis study approximately 5 days after surgery. In studies M2 and M4, mice were implanted with $1 \times 10^{5}$ mouse G3MB cells and cannulas simultaneously under a previously described Institutional Animal Care and Use approved procedure. The stereotaxic coordinates for cannula implantation were: $\mathrm{x}=-1 \mathrm{~mm}, \mathrm{y}=-2 \mathrm{~mm}$, and $\mathrm{z}=$ $2.5 \mathrm{~mm}$ using the bregma as the zero point. Tumor growth was measured weekly by bioluminescence imaging using a Xenogen (Caliper Life Science, Waltham, MA, USA) system. After the tumor cells grew for $\sim 14$ days and bioluminescence signals reached $\sim 1 \times 10^{8}$ photons $/ \mathrm{sec}$, mice were enrolled in the microdialysis study. For all mice, on the day of microdialysis experiment, the stylet was removed from the cannula and a 1-mm microdialysis probe with $38 \mathrm{kDa}$ molecular weight cut-off membrane (MD-2211, BASi) was inserted into the guide cannula to collect brain/tumor extracellular fluid (ECF). The probes were perfused with Ringer's solution at a flow rate of $0.5 \mu \mathrm{L} / \mathrm{min}$ for $1 \mathrm{~h}$ to equilibrate with the in vivo environment. Once the probes were equilibrated, mice were dosed with 130 $\mathrm{mg} / \mathrm{kg}$ cyclophosphamide via IP injection. Dialysate fractions containing unbound drug ECF cyclophosphamide, 4OH-CTX, and CEPM concentrations were collected over $1 \mathrm{~h}$ intervals for $5 \mathrm{~h}$.

Similar to the plasma pharmacokinetic study, a derivatizing solution was added to the dialysate samples to stabilize and analyze 4OH-CTX. The derivatizing solution was either directly added to the perfusate (i.e., derivatized Ringer's solution) as in studies M1 and M2, or placed into the tubes in which dialysates were collected as in studies M3 and M4. During the four cerebral microdialysis studies, blood samples from each mouse were collected at time 
points as defined by a limited sampling model (see Pharmacokinetic Modeling) by retro-orbital bleeds, and processed as previously described. All plasma and dialysate samples were stored at $-80^{\circ} \mathrm{C}$ until further analysis. As quality control measure, after completion of the microdialysis studies, the brain was removed with the microdialysis probe intact, and submitted for routine processing and histology. Brains were immersion fixed in situ in 10\% neutral buffered formalin for a minimum of $24 \mathrm{~h}$ before removal from the calvarium, then were fixed for an additional $24 \mathrm{~h}$. The brains were embedded in paraffin, $4 \mu \mathrm{m}$ thick horizontal sections were taken at $150 \mu \mathrm{m}$ intervals and were stained with hematoxylin and eosin (HE).The slides were examined to confirm the location of the probe within the tumor (for tumor-bearing animals) and detect any hemorrhage or necrosis.

\section{Recovery studies}

The dialysate flowing through the probe and the ECF surrounding the probe are not in equilibrium with one another. Thus, recovery studies must be performed for each probe prior to its use in the microdialysis study to relate dialysate results to the drug concentrations in tumor or brain ECF (19). For this analysis, microdialysis recovery was determined using in vitro retrodialysis recovery studies. For in vitro retrodialysis, each probe for each non-tumor and tumor-bearing mouse was placed in a stirred blank Ringer's solution and maintained at $37^{\circ} \mathrm{C}$. The probe was either perfused with derivatized Ringer's solution or blank Ringer's solution with the derivatizing solution in the collection tube at a known concentration of the drug of interest $(1 \mu \mathrm{g} / \mathrm{mL}$ for each cyclophosphamide, 4OH-CTX, and CEPM) at a flow rate of $0.5 \mu \mathrm{L} / \mathrm{min}$. After equilibration for $1 \mathrm{~h}$, three consecutive fractions of $1 \mathrm{~h}$ interval each were collected. Dialysate samples were stored at $80^{\circ} \mathrm{C}$ until further analysis. For each compound, probe recovery was calculated using the ratio of drug concentration in the dialysate sample to that in the bulk sample. Unbound concentration in tumor or brain ECF was then calculated by dividing concentration observed in dialysate samples collected during the microdialysis study with the in vitro recovery ratios determined with the appropriate probe for each compound in each mouse.

Prior to perform the cerebral microdialysis studies, the concentration-dependency of the in vitro recovery was evaluated using three different cyclophosphamide concentrations, i.e., 20, 200, and $2000 \mathrm{ng} / \mathrm{mL}$ and four different probes with three replicates per probe. The average recovery coefficients obtained for each probe at each concentration were compared to assess the recovery concentration-dependency using one-way ANOVA with the Geisser-Greenhouse correction and Tukey's multiple comparison tests.

\section{Bioanalytical method}

The LC-MS/MS methods to analyze human plasma cyclophosphamide and its metabolites samples previously developed by Kalhorn and colleagues were modified and validated to analyze cyclophosphamide and CEPM in one method, and derivatized 4OH-CTX in another method with adequate precision and bias $(16,17)$. The details of the modifications of the LC-MS/MS methods are reported in Supplementary Material (Section 1). The lower limits of quantification (LLOQ) for cyclophosphamide, 4OH-CTX and CEPM in plasma were $0.038,0.017$, and $0.20 \mu \mathrm{M}$, respectively. The LLOQ for cyclophosphamide, 4OH-CTX, and CEPM in Ringer's were $0.0038,0.0068$, and 0.020 $\mu \mathrm{M}$, respectively.

\section{Pharmacokinetic modeling General methods}

Plasma and ECF concentration-time data for cyclophosphamide, 4OH-CTX, and CEPM were analyzed with a population pharmacokinetic approach using non-linear mixed effect modeling with Monolix v2018R1 (Antony, France: Lixoft SAS, 2018; http://lixoft.com/products/monolix). Population parameters were estimated with the Stochastic Approximation Expectation Maximization algorithm. Inter-individual terms associated with pharmacokinetic parameters were assumed to follow a log-normal distribution. For parent drug and metabolites, plasma and ECF models, different model structures were tested (e.g., one or two-compartment models, linear or non-linear elimination), and additive and/or proportional residual variability models were applied. Plasma and ECF concentration-time data below LLOQ were included in the model dataset as censored (20). Model selections were based upon the goodness-of fit plots (21), precision of parameter estimates (relative standard errors; RSE\%), and change in the maximization of the likelihood expressed as the objective function value. The final models were 
evaluated using visual predictive checks (22). Simulations of 500 replicates of the datasets were performed. The observed data (i.e., plasma or ECF concentrations) were overlaid on the $5^{\text {th }}, 50^{\text {th }}$, and $95^{\text {th }}$ percentiles of the model simulations to visually assess concordance between observations and model simulations. GraphPad Prism (version 8.1 for Windows, www.graphpad.com) was used for statistical analyses and graphical presentations.

\section{Modeling strategy:}

A stepwise modeling strategy was used. First, cyclophosphamide, 4OH-CTX, and CEPM plasma concentrations obtained from the plasma pharmacokinetic study were simultaneously modeled to characterize the plasma disposition of each compound. A limited sampling model was developed for the subsequent microdialysis studies based on the parameter estimates of cyclophosphamide, the primary drug of interest. Due to limitations upon total blood volume that could be withdrawn from each mouse and the volume of plasma required for bioanalysis, the microdialysis studies were limited to three plasma samples per mouse. The limited sampling model was developed using the D-optimality method implemented in ADAPT 5 (BSMR, Los Angeles, CA, USA) to derive statistically informative time points for plasma sample collection $(23,24)$.

Second, the cyclophosphamide, $4 \mathrm{OH}-\mathrm{CTX}$, and CEPM plasma data collected during the microdialysis studies were combined with those from the previous plasma study, and the plasma model parameters were re-estimated. Individual post-hoc plasma pharmacokinetic parameters were obtained for each mouse included in the microdialysis studies and were used to characterize the unbound tumor/brain ECF disposition of each compound. ECF concentrations were modeled using one or two-compartments linked to the central plasma compartment. Only free cyclophosphamide, 4OH-CTX, and CEPM were assumed to cross the blood-brain-barrier or brain-tumor-barrier (25). For each compound, only unbound drug was assumed to be distributed into the ECF compartment, thus the amount of drug in the plasma was multiplied by the corresponding $\mathrm{F}_{\mathrm{U}}$. The drug ECF concentrations collected during the microdialysis studies were simultaneously analyzed.

For each mouse, model-derived area under plasma and unbound tumor/brain ECF concentration-time curve $\left(\mathrm{AUC}_{\mathrm{P}, 0-5 \mathrm{~h}}\right.$ and $\mathrm{AUC}_{\mathrm{ECF}, 0-}$ ${ }_{5 \mathrm{~h}}$ ) were generated for each compound by integration of the plasma and ECF concentration-time profiles from time zero to $5 \mathrm{~h}$. The plasma exposure values of unbound cyclophosphamide, 4OH-CTX, and CEPM $\left(\mathrm{AUC}_{\mathrm{u}, \text { plasma }}\right)$ were derived as the product of each compound $\mathrm{AUC}_{\mathrm{P}, 0-5 \mathrm{~h}}$ and $\mathrm{F}_{\mathrm{U}}$. The $\mathrm{CNS}$ penetration of each drug in both non-tumor- and tumor-bearing mice was assessed by calculating the unbound tumor/brain to plasma partition coefficient $\left(\mathrm{K}_{\mathrm{p}, \mathrm{uu}}\right)$ as follows:

$$
K_{p, u u}=\frac{A U C_{E C F}}{A U C_{u, p l a s m a}}
$$

The model-derived drug exposures in plasma, tumor, and brain, as well as the $\mathrm{K}_{\mathrm{p} \text {,u }}$ values were statistically compared between the non-tumor- and tumor-bearing mice using two-tailed unpaired t-test with Welch's correction. A p-value of $\leq 0.05$ was considered significant.

\section{RESULTS}

\section{Plasma protein binding studies}

The protein binding of cyclophosphamide, $4 \mathrm{OH}-$ CTX, and CEPM in CD1 murine plasma was assessed by subjecting spiked plasma samples to rapid equilibrium dialysis. The compounds were negligibly bound to the dialysis membrane contained in the Centrifree ${ }^{\circledR}$ ultrafiltration devices, suggesting that rapid equilibrium dialysis was a suitable technique for determining cyclophosphamide and metabolites protein binding. The median (range) $\mathrm{F}_{\mathrm{U}}$ values of cyclophosphamide, $4 \mathrm{OH}-\mathrm{CTX}$, and CEPM in mouse plasma were $0.26(0.24-0.28), 0.39$ (0.28$0.48)$, and $0.31(0.29-0.34)$, respectively.

\section{Plasma pharmacokinetics}

The characteristics of the plasma study performed in non-tumor-bearing mice are presented in Table 1. The cyclophosphamide data below the LLOQ represented $40 \%$ of all collected data, with no cyclophosphamide concentration measurable from $4 \mathrm{~h}$ post-dose. Only $10 \%$ of the $4 \mathrm{OH}-\mathrm{CTX}$ data fell below the LLOQ, while all the CEPM data were measurable up to $6 \mathrm{~h}$ post-dose. Plasma cyclophosphamide, 4OH-CTX, and CEPM data were each well-fitted with a two-compartment linear model (Fig. 1). For identifiability issues, cyclophosphamide and 4OH-CTX were assumed to 
be fully converted into 4OH-CTX and CEPM, respectively. Thus, the clearance and volume parameters estimated for 4OH-CTX and CEPM were apparent.

Table 1. Pharmacokinetic studies characteristics

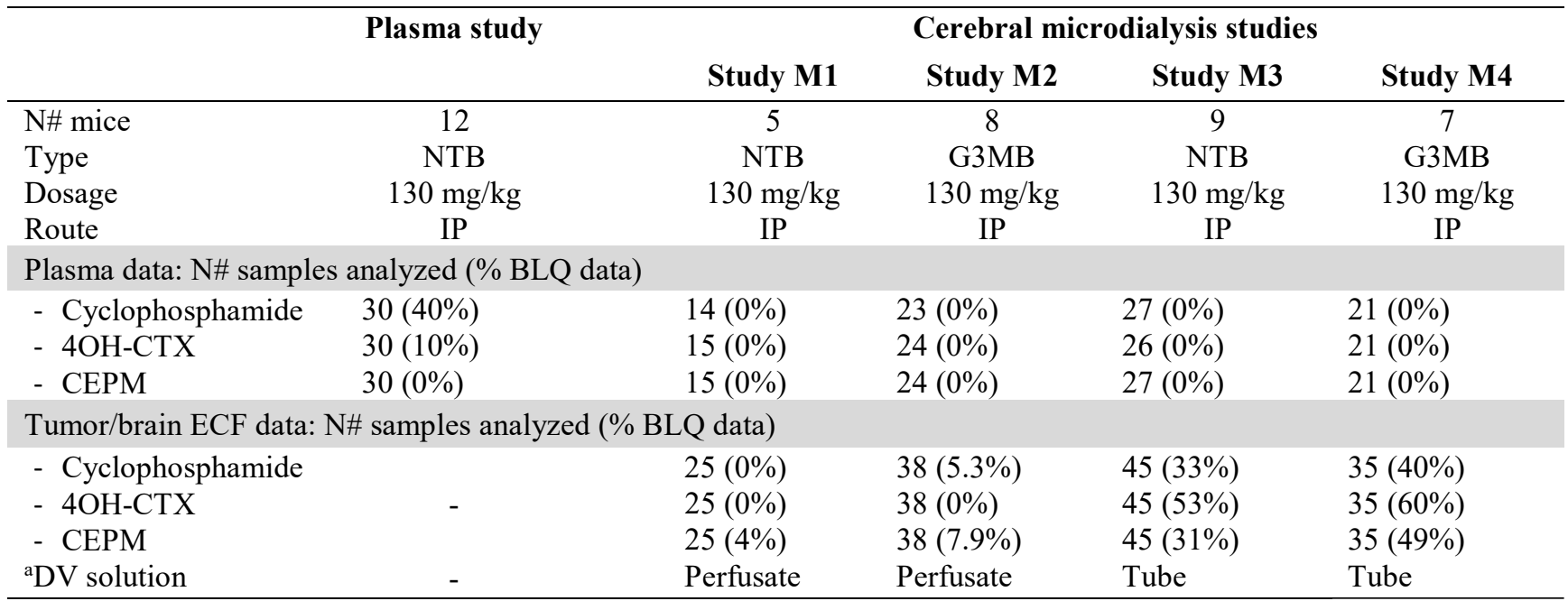

NTB non-tumor bearing mice, G3MB mice orthotopically implanted with Group 3 medulloblastoma, BLQ below the limit of quantification, ECF extracellular fluid, 4OH-CTX 4-hydroxy cyclophosphamide, CEPM carboxyethylphosphoramide mustard, DV derivatizing solution to stabilize 4OH-CTX, IP intraperitoneal. ${ }^{a}$ A phenylhydrazine derivatizing (DV) solution was added to the collected plasma and ECF samples to stabilize and analyze 4OH-CTX. For plasma samples, the DV solution was added in the collection tubes after blood sampling. For ECF samples, the DV solution was either added in the perfusate in the microdialysis probe, or added in the dialysate collection tubes.

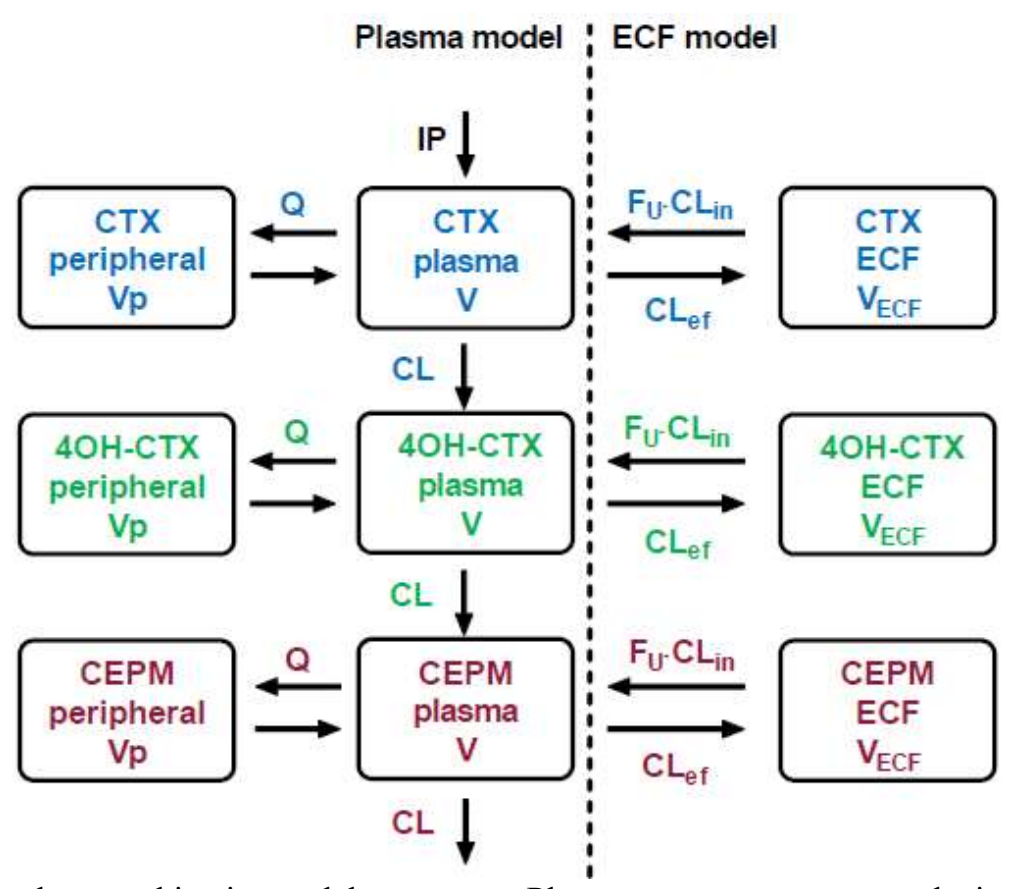

Figure 1. Plasma-ECF pharmacokinetic model structure. Plasma compartments are depicted on the left, and ECF compartments are depicted on the right. All blue compartments represent cyclophosphamide (CTX), green compartments represent 4-hydroxy-cyclophosphamide (4OH-CTX) and red compartments represent carboxyethylphosphoramide mustard (CEPM). For each compound, "CL", "V", "Q" and "Vp" are the central clearance, central volume, peripheral clearance and peripheral volume of distribution, respectively. $\mathrm{F}_{\mathrm{U}}$ is the fraction unbound in plasma. " $\mathrm{V}_{\mathrm{ECF}}$ " is the volume of the ECF central compartment, and $\mathrm{CL}_{\mathrm{in}}$ and $\mathrm{CL}_{\mathrm{ef}}$ are the influx and efflux clearances. 
The mean plasma pharmacokinetic parameters of cyclophosphamide were used to derive a limited sampling model for use in the subsequent microdialysis studies. The most informative timepoints were determined at $0.25,1$, and $2 \mathrm{~h}$ post-dose.

The plasma data collected during the microdialysis studies were combined with the data from the plasma pharmacokinetic study, and the model parameters were re-estimated. The final parameters were estimated with good precision (all $\mathrm{RSE}<30 \%$ ), and are reported in Table 2. The diagnostic plots for the plasma model are depicted in Supplementary Material (Fig. S1). The model VPCs are presented in Fig. 2 ( $1^{\text {st }}$ column). The central tendency and variability of cyclophosphamide, 4OH-CTX, and CEPM plasma concentrations in the mouse population were well predicted by the selected model. Mean observed maximum concentrations were $352.9,81.9$, and 84.8 $\mu \mathrm{M}$ for cyclophosphamide, 4OH-CTX, and CEPM, respectively. Mean model-derived plasma exposures of total cyclophosphamide, 4OH-CTX, and CEPM $\left(\mathrm{AUC}_{\mathrm{P}, 0-5 \mathrm{~h}}\right)$ in mice were estimated at 112.6, 45.6, and $80.8 \mu \mathrm{M} \cdot \mathrm{h}$, respectively.

CNS penetration of cyclophosphamide and its metabolites

Concentration-independency of the in vitro recovery

The concentration-dependency of the in vitro recovery was evaluated using different cyclophosphamide concentrations. The average recovery values obtained for the following cyclophosphamide concentrations: 20, 200, and $2000 \mathrm{ng} / \mathrm{ml}$ were $15.3 \%, 27.2 \%$, and $11.8 \%$, respectively $(\mathrm{p}>0.5)$. The individual recovery values are depicted in Supplementary Material (Fig. S2).

Table 2. Final pharmacokinetic parameter estimates for cyclophosphamide and its metabolites

\begin{tabular}{|c|c|c|c|}
\hline \multirow[b]{2}{*}{ Parameter (Unit) } & \multicolumn{3}{|c|}{ Estimate (RSE\%) } \\
\hline & Cyclophosphamide & 4ОН-СТX & CEPM \\
\hline \\
\hline \multicolumn{4}{|l|}{ Population estimates } \\
\hline Central clearance CL (L/h/kg) & $4.4(3.7)$ & $11(4.9)$ & $6.4(3.0)$ \\
\hline Central volume V (L/kg) & $0.65(5.0)$ & $2.4(6.2)$ & $1.1(5.3)$ \\
\hline Peripheral clearance $\mathrm{Q}(\mathrm{L} / \mathrm{h} / \mathrm{kg})$ & $0.18(4.3)$ & $0.075(17)$ & $1.6(9.0)$ \\
\hline Peripheral volume Vp (L/kg) & $0.062(2.6)$ & $0.22(20)$ & $1.7(7.1)$ \\
\hline Proportional residual variability $\varepsilon_{\text {prop }}$ & $0.24(11)$ & $0.29(11)$ & $0.18(12)$ \\
\hline \multicolumn{4}{|l|}{ Inter-individual variabilities (IIV) } \\
\hline Central clearance $\eta-C L$ & $0.11(17)$ & $0.13(19)$ & $0.094(25)$ \\
\hline Central volume $\eta-\mathrm{V}$ & - & - & $0.22(21)$ \\
\hline \multicolumn{4}{|l|}{ ECF MODEL } \\
\hline \multicolumn{4}{|l|}{ Population estimates } \\
\hline Influx clearance $\mathrm{CL}_{\text {in }}(\mathrm{L} / \mathrm{h} / \mathrm{kg})$ & $4.3 \cdot 10^{-4}(16)$ & $2.3 \cdot 10^{-4}(25)$ & $8.8 \cdot 10^{-5}(17)$ \\
\hline Efflux clearance $\mathrm{CL}_{\mathrm{ef}}(\mathrm{L} / \mathrm{h} / \mathrm{kg})$ & $2.4 \cdot 10^{-3}(7.0)$ & $3.3 \cdot 10^{-3}(4.9)$ & $1.5 \cdot 10^{-3}(7.2)$ \\
\hline Proportional residual variability $\varepsilon_{\text {prop }}$ & $0.45(12)$ & $0.33(16)$ & $0.22(15)$ \\
\hline \multicolumn{4}{|l|}{ Inter-individual variabilities (IIV) } \\
\hline Influx clearance $\eta-C L_{\text {in }}$ & $0.31(51)$ & $0.86(19)$ & $0.62(19)$ \\
\hline Efflux clearance $\eta-C L_{e f}$ & $0.23(22)$ & - & $0.20(38)$ \\
\hline
\end{tabular}

CTX, cyclophosphamide, 4OH-CTX 4-hydroxy cyclophosphamide, CEPM carboxyethylphosphoramide mustard, RSE\% relative standard errors, ECF extracellular fluid.

Inter-individual variabilities are reported in terms of standard deviations. All inter-individual terms were assumed to follow a log-normal distribution as follows: $P_{i}=\theta_{P_{i}} \cdot e^{\eta_{P_{i}}}$ where $\mathrm{P}_{\mathrm{i}}$ is the pharmacokinetic parameter for the $\mathrm{i}^{\text {th }}$ individual, $\theta_{\mathrm{pi}}$ the population typical value for $\mathrm{P}_{\mathrm{i}}$, and $\eta_{\mathrm{p}}$ the inter-individual variability effect. 

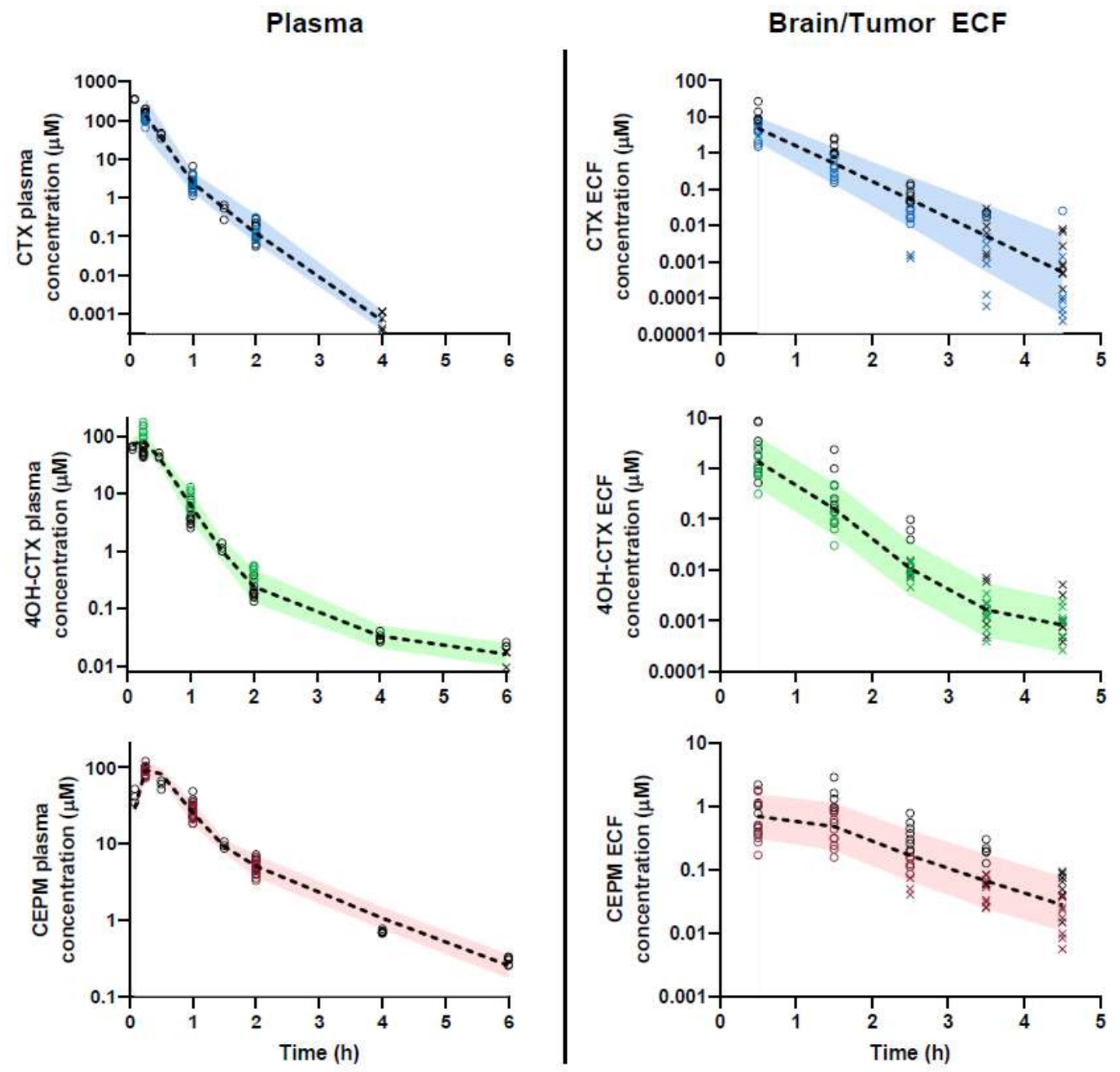

Figure 2. Observed data and visual predictive checks (VPC) for plasma and ECF cyclophosphamide and metabolites concentration-time data. The first column shows the observed data and model VPC for plasma cyclophosphamide CTX (blue), 4-hydroxy-cyclophosphamide 4OH-CTX (green) and carboxyethylphosphoramide mustard CEPM (red). The second column shows the observed data and model VPC for brain or tumor brain extracellular fluid CTX (blue), 4OH-CTX (green) and CEPM (red). In each graph, black open-circles represent the observed concentrations in non-tumor bearing mice, and colored open-circles are the observed concentrations in mice bearing orthotopic Group 3 medulloblastoma. The crosses represent the simulated data below the limit of quantification. Dashed lines depict the mean (50 ${ }^{\text {th }}$ percentile) of model simulated concentrations, and the shaded areas represent the $90 \%$ prediction interval $\left(5-95^{\text {th }}\right.$ percentiles) of the model simulations.

The results showed that the recovery was concentration independent. Therefore, one concentration (i.e., $1 \mu \mathrm{g} / \mathrm{ml}$ ) was used for the subsequent in vitro recovery studies during the cerebral microdialysis.

\section{Impact of different methods to collect $4 \mathrm{OH}-\mathrm{CTX}$ samples in ECF}

Cerebral microdialysis was used to determine both mouse brain and tumor ECF disposition of cyclophosphamide and its metabolites after a dosage of $130 \mathrm{mg} / \mathrm{kg}$ IP. The bioanalytical analysis of the collected data revealed large differences between the studies M1 and M2 with the derivatizing solution in the microdialysis probe perfusate, $v s$ the studies M3 and M4 with the derivatizing solution added in the dialysate collection tubes. In studies M1 and M2, most of the cyclophosphamide, 4OH-CTX, and CEPM collected data were quantifiable for up to $5 \mathrm{~h}$ post-dose (data not shown). However, in studies M3 
and M4, most of the cyclophosphamide, 4OH-CTX, and CEPM concentrations fell below the LLOQ after $3 \mathrm{~h}$ post-dose.

The histology studies performed with HE staining to confirm the location of the microdialysis probes confirmed a difference between the two approaches used to stabilize $4 \mathrm{OH}-\mathrm{CTX}$. In the studies M1 and M2 with the derivatizing solution in the probe, the brain tissues exhibited hemorrhage and necrosis which represented on average $18 \pm 20 \%$ and $17 \pm 5 \%$ of the areas examined, respectively. In comparison, in the studies with the derivatizing solution in collection tubes, only $1.0 \pm 1.2 \%$ and $2.6 \pm 0.9 \%$ of the brain sections examined were hemorrhagic and necrotic, respectively. As an example, Fig. 3 shows representative sections of brain tissues of two non-tumor-bearing mice; one with derivatizing solution in the probe (right) and one with derivatizing solution in the collection tubes (left). The presence of blood around the membrane may have led to a contamination of the ECF collected samples in studies M1 and M2, which could explain the higher observed concentrations for cyclophosphamide, 4OH-CTX, and CEPM. In consequence, the data collected in studies M1 and M2 were excluded from further analysis.

\section{Tumor and brain ECF pharmacokinetic modeling}

The cyclophosphamide, 4OH-CTX, and CEPM data collected from the studies M3 and M4 were corrected by the individual calculated in vitro recovery coefficients. Mean ( \pm SD) obtained recovery coefficients for cyclophosphamide, 4OH-CTX, and CEPM were $16.8 \%(6.2), 28.1 \%(8.1)$, and $18.0 \%$ (4.8) in study M3, and 29.2\% (4.3), 45.6\% (4.1), and $25.4 \%$ (4.2) in study M4. The ECF concentrations were then simultaneously modeled with the plasma concentration-time profiles previously derived in each mouse. Tumor and brain ECF concentrationtime data were well fitted by a one-compartment model for cyclophosphamide, 4OH-CTX and CEPM, parameterized with influx and efflux clearances (Fig. 1). To avoid over-parameterization, only the transfer of each compound between plasma and ECF compartments were modeled. The metabolic processes occurring in the brain were assumed negligible compared to those happening in liver. Lastly, the volume of each ECF central compartment was fixed to $0.001 \mathrm{~L} / \mathrm{kg}$ based on published data (26). The ECF pharmacokinetic parameters estimated in mice enrolled in studies M3M4 are reported in Table 2. As shown in Fig. 2 and Fig. S3, the model was able to capture the central tendency and the variability of cyclophosphamide and its metabolites ECF concentrations.

The model-derived unbound plasma exposures, unbound tumor/ brain ECF exposures, and $\mathrm{K}_{\mathrm{p} \text {,uu }}$ values for cyclophosphamide, 4OH-CTX, and CEPM in both non-tumor and tumor bearing mice are reported in Table 3. The $\mathrm{K}_{\mathrm{p} \text {,uu }}$ values for each compound were compared between the two groups of mice: non-tumor-bearing mice $v s$ mice bearing orthotopic G3MB.

\section{A] DV solution in collection tube}

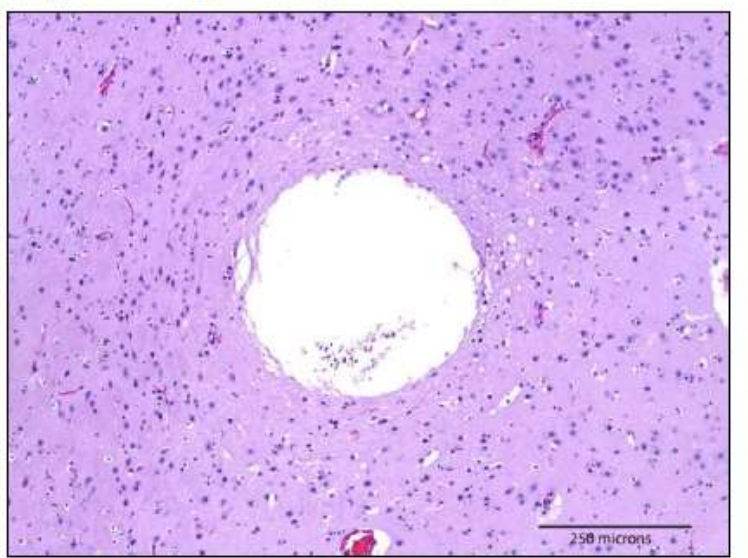

B] DV solution in perfusate

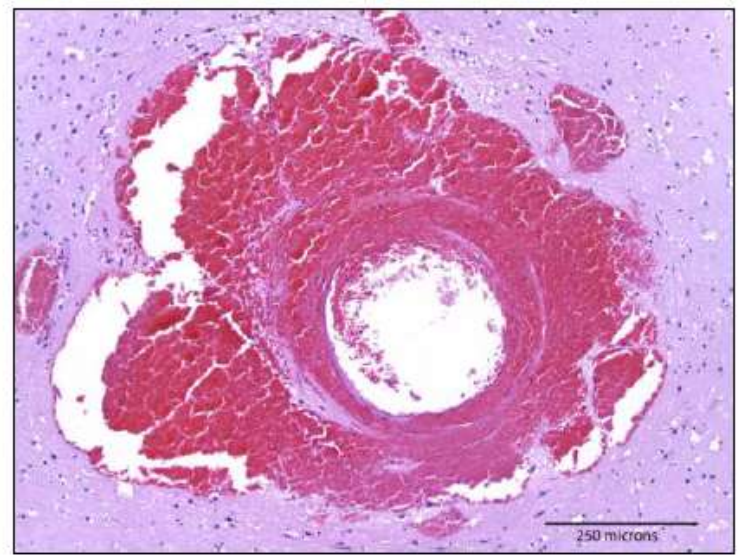

Figure 3. Representative sections of microdialysis probe locations in a non-tumor bearing mouse brain for which no derivatizing solution was added in the perfusate (A), and in a non-tumor-bearing mouse brain for which the derivatizing solution was added in the perfusate (B). Tissue was harvested 24 hours after treatment and stained with hematoxylin and eosin. Scale equals to $250 \mu \mathrm{m}$. Large lake of hemorrhage surrounding the membrane where the probe was located was observed in the mouse brain which had the derivatizing solution in the perfusate. 
Table 3. Model-derived unbound plasma and ECF exposures $(\mu \mathrm{M} \cdot \mathrm{h})$ and ECF to plasma partition coefficients $\left(\mathrm{K}_{\mathrm{p}, \mathrm{uu}}\right)$ for cyclophosphamide and its metabolites.

\section{NTB bearing mice (Study G3MB bearing mice (Study $\quad$ All mice (Studies M3-M4)}

M3) M4)

\begin{tabular}{|c|c|c|c|}
\hline \multicolumn{4}{|l|}{ AUC $\mathbf{u}_{u, p l a s m a, 0-5 h}$} \\
\hline - CTX & $29.2(3.6)$ & $27.9(3.1)$ & $28.6(3.3)$ \\
\hline - $4 \mathrm{OH}-\mathrm{CTX}$ & $15.6(0.7)$ & $20.2(0.8)$ & $17.8(2.5)$ \\
\hline - CEPM & $25.1(1.6)$ & $23.1(1.7)$ & $24.2(1.9)$ \\
\hline \multicolumn{4}{|l|}{$A U C_{E C F, 0-5 h}$} \\
\hline - CTX & $6.3(1.3)$ & $4.0(1.4)$ & $5.2(1.8)$ \\
\hline - 4OH-CTX & $2.1(2.1)$ & $1.0(0.7)$ & $1.6(1.7)$ \\
\hline - CEPM & $2.1(0.9)$ & $1.0(0.7)$ & $1.6(0.9)$ \\
\hline \multicolumn{4}{|l|}{$\mathbf{K}_{\mathrm{p}, \mathrm{uu}}$} \\
\hline$-\mathrm{CTX}^{* *}$ & $0.22(0.05)$ & $0.15(0.05)$ & $0.18(0.06)$ \\
\hline - 4OH-CTX & $0.14(0.14)$ & $0.05(0.03)$ & $0.10(0.11)$ \\
\hline CEPM & $0.08(0.04)$ & $0.05(0.03)$ & $0.07(0.04)$ \\
\hline
\end{tabular}

Data are reported in terms of mean (standard deviation).

NTB, non-tumor-bearing; G3MB, Group 3 medulloblastoma

$\mathbf{A U C}_{\mathbf{u}, \text { plasma,0-5h }}$ are the unbound plasma drug exposures. They were calculated as the product of each compound total plasma exposure $\left(\mathrm{AUC}_{\mathrm{P}, 0-5 \mathrm{~h}}\right)$ derived by the plasma pharmacokinetic model, and plasma fractions unbound $\mathrm{F}_{\mathrm{U}}$ determined by equilibrium dialysis.

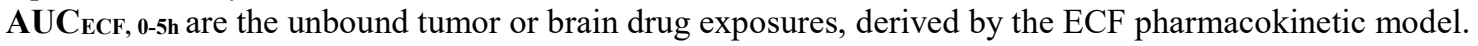

$\mathbf{K}_{\mathbf{p} \text {,uu }}$ are the unbound tumor or brain ECF to plasma partition coefficients. They were calculated as the ratio between $\mathrm{AUC}_{\mathrm{ECF}, 0-5 \mathrm{~h}}$ and $\mathrm{AUC}_{\mathrm{u}, \mathrm{plasma}, 0-5 \mathrm{~h}}$ for each compound.

**Unpaired t-tests with Welch's correction were performed to compare the $\mathrm{K}_{\mathrm{p}, \mathrm{uu}}$ values between the non-tumor bearing mice and mice orthotopically implanted with G3MB. Significantly lower $\mathrm{K}_{\mathrm{p} \text {,u }}$ values were observed for cyclophosphamide in tumor-bearing mice compared to non-tumor bearing mice ( $\mathrm{p}$-value $=0.019)$. No differences were found for the two metabolites $\mathrm{K}_{\mathrm{p} \text {,uu. }}$.

A significantly lower cyclophosphamide CNS penetration was observed in G3MB mice (pvalue $=0.019$ ) compared to non-tumor-bearing mice. No significant differences in CNS penetration of 4OH-CTX and CEPM were observed between nontumor- and tumor-bearing mice.

\section{DISCUSSION}

The overall objective of the study was to evaluate the penetration of cyclophosphamide and its metabolites into the brain and brain tumors for treatment of pediatric medulloblastoma. Understanding the extent by which a drug crosses the blood-brain barrier and enters brain tumors is crucial as this will affect the clinical efficacy of the drug. Cyclophosphamide in combination with other chemotherapeutic agents has become as standard of care for the treatment of pediatric primary brain tumors. As such, it has been used in efficacy studies in mouse models of medulloblastoma to investigate new combination treatments. Despite its long-term use, limited information about cyclophosphamide ability to cross the blood-brain barrier is available. To our knowledge, no studies have investigated the penetration of cyclophosphamide and/or its metabolites into the mouse brain. Based upon the survival advantage that cyclophosphamide as a single agent or in combination confers preclinically and clinically $(7,9)$, its CNS penetration may be assumed to be adequate for efficacy. However, the characterization of cyclophosphamide and/or its metabolite pharmacokinetics in the brain and brain tumor provide valuable information which will help to establish exposure-response relationships in pediatric medulloblastoma. In preclinical studies, this will further help to evaluate and optimize new combination therapies to guide the design of future clinical trials.

This perspective motivated our current analysis which focused on the CNS penetration of cyclophosphamide, 4OH-CTX, and CEPM using a mouse model of pediatric G3MB, a subgroup of medulloblastoma with poor prognosis (4). It also used non-tumor-bearing mice to completely evaluate the brain penetrance of the drug, and identify what impact the presence of a tumor has on drug CNS penetration (27). The preclinical approach used in 
this present study involves both systemic and CNS tumor pharmacokinetic studies with populationbased pharmacokinetic modeling and simulation (18). The inactive metabolite CEPM was included in the study, as it can be simultaneously analyzed with cyclophosphamide with the same bioanalytical method. Furthermore, the inclusion of a downstream metabolite was thought to help in estimating the pharmacokinetic parameters for 4OH-CTX.

Cyclophosphamide, 4OH-CTX, and CEPM plasma concentration-time data were adequately described simultaneously by two-compartment models, and no differences were observed between non-tumor-bearing and tumor-bearing mice. All three compounds were rapidly eliminated from plasma, specifically cyclophosphamide with no concentration measurable by 4 hours post-dose. The ratios of $4 \mathrm{OH}-\mathrm{CTX}$ and CEPM plasma AUC to cyclophosphamide plasma AUC, 0.40 and 0.72, respectively, were much greater than those that have been observed in humans (28-30). Cyclophosphamide is known to undergo extensive metabolism, controlled by multiple cytochrome P450 enzymes among others (15). Several isoforms of P450 enzymes have been shown interspecies differences in terms of activity; thus, it was not unexpected to observe different metabolic behavior in the mouse model (31). Another noticeable difference between mice and humans was the extent of cyclophosphamide plasma protein binding. Our study showed a higher fraction unbound for the parent drug $\left(\mathrm{F}_{\mathrm{U}}=0.26\right)$ compared to what has been measured in humans $\left(\mathrm{F}_{\mathrm{U}} 7-17 \%\right)$. However, the $\mathrm{F}_{\mathrm{U}}$ values determined for the two metabolites $(4 \mathrm{OH}-$ $\mathrm{CTX} \mathrm{F}_{\mathrm{U}}=0.39$ and $\mathrm{CEPM} \mathrm{F}_{\mathrm{U}}=0.31$ ) were similar to what has been observed clinically $(15,32)$.

When performing microdialysis studies, recovery studies are required to estimate the fraction of the drug recovered through the probe and calculate the "true" tissue concentration. The recovery coefficients can be determined using several methods such as in vitro dialysis, in vitro retrodialysis and in vivo retrodialysis which obviously should be preferred (19). Both in vivo and in vitro retrodialysis were performed during our cerebral microdialysis studies for cyclophosphamide and its metabolites. Similar in vivo and in vitro recovery values were observed for some probes; however, several negative in vivo recovery values were obtained in other probes. Therefore, the in vivo recovery values could not be used in these studies, and the conservative in vitro approach was used to calculate the brain and tumor ECF concentrations.

As a putative active metabolite, $4 \mathrm{OH}-\mathrm{CTX}$ is important to characterize, although its bioanalysis requires the addition of a derivatizing solution to the collected samples immediately after sampling. During the microdialysis process, this could be done in two different ways. The derivatizing solution could be added in the perfusate, which would prevent from any delays between the collection of the sample and the stabilization of $4 \mathrm{OH}-\mathrm{CTX}$. However, the derivatizing solution would be in direct contact with brain tissue. Alternatively, the solution could be added into the tubes in which the ECF samples were collected, without contact with brain tissue but with potential delay to stabilize 4OH-CTX. Both methods were tested in non-tumor-bearing and tumor-bearing mice. The results clearly highlighted a negative effect of the derivatizing solution placed in the dialysate (Fig. 3). First, evidence of tissue necrosis and hemorrhage surrounding the probe location was observed. Then, the ECF concentration-time profiles obtained in these studies (M1 and M2) for the three compounds differed from those obtained in studies M3 and M4 with solution in collection tubes. These results led us to stop using the derivatizing solution into the probe for any future study.

Overall, mice exhibited large inter-individual variability in the ECF concentration-time profiles for cyclophosphamide, 4OH-CTX, and CEPM. ECF concentrations for the three compounds were measurable for a few hours after dose administration before falling below the limit of quantification. Unbound brain or tumor to plasma partition

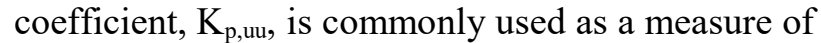
the extent of drug distribution into the tissue (brain or tumor). On average, cyclophosphamide, $4 \mathrm{OH}-$

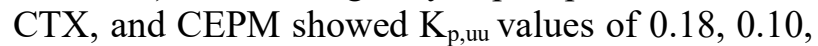
and 0.07 , respectively, in both non-tumor and tumorbearing mice (Table 3). The model-derived $\mathrm{K}_{\mathrm{p} \text {,u }}$ values for cyclophosphamide highlighted significant differences between non-tumor-bearing mice and those orthotopically implanted with G3MB. Cyclophosphamide exhibited lower CNS penetration in presence of tumor compared to normal brain (mean $\mathrm{K}_{\mathrm{p} \text {,u }} 0.15$ for tumor-bearing mice versus 0.22 for non-tumor bearing mice). This result is not completely understood and needs to be interpreted carefully considering the high inter-subject variability in the ECF observed concentrations, the small number of animals included in the studies, and 
the non-significant different extents of CNS penetration of the metabolites. One explanation is higher efflux transport at the blood-brain tumor barrier compared to that occurring at the blood-brain barrier since cyclophosphamide is a substrate for several transporters, such as P-glycoprotein (ABCB1) expressed in the mouse model of G3MB $(33,34)$. Another potential explanation would be a more extensive drug metabolism at the blood-brain tumor barrier compared to that occurring at the blood-brain-barrier, although no significant differences were observed in the CNS penetration of the metabolites.

To our knowledge, no $\mathrm{IC}_{50}$ value or minimum effective concentration have been reported for the active metabolite 4OH-CTX in Group 3 medulloblastoma. Friedman and colleagues found that a range of cyclophosphamide concentrations between 4.7 and $6.6 \mu \mathrm{M}$ would lead to $1-\log$ kill in several medulloblastoma cell lines in vitro (35). These values are in the range of the maximum ECF concentrations we observed although the comparison should be interpreted with caution as these values were obtained in different medulloblastoma cell lines than the one used in this analysis. The dosage used in our studies (130 mg/kg cyclophosphamide) was selected based on previously published studies which demonstrated efficacy against medulloblastoma mouse models $(9,10)$. White and Sterling-Levis used an intraocular xenograft model of medulloblastoma/primitive neuroectodermal tumor (cell line JRMB-6) to study components of the VETOPEC (vincristine, etoposide, cyclophosphamide) multiagent chemotherapy regimen (9). When administrated as a single agent at $130 \mathrm{mg} / \mathrm{kg}$ IP daily for five consecutive days, it produced an $85 \%$ response rate and statistically improved survival versus saline controls. The same cyclophosphamide dosage regimen was further investigated in combination with cisplatin (IV, 5 $\mathrm{mg} / \mathrm{kg}$ at day 1) in mice orthotopically implanted with G3MB by Morfouace and colleagues (10). Mice treated with cisplatin and cyclophosphamide (IP, 130 $\mathrm{mg} / \mathrm{kg}$, days 2-6) exhibited a median survival of 12 days longer compared to the vehicle-treated mice. The population plasma-ECF model was used to simulate the cumulative ECF exposures of cyclophosphamide, 4OH-CTX, and CEPM after the same dosing regimen in tumor-bearing mice (130 $\mathrm{mg} / \mathrm{kg}$, IP 5-days). Assuming that the CNS penetration of the compounds was linear after repeated doses, the mean cumulative ECF exposures were $27.6,5.3$, and $8.9 \mu \mathrm{M} \cdot \mathrm{h}$ for cyclophosphamide, 4OH-CTX, and CEPM, respectively based on 500 simulations (Supplementary Material Figure S4). Thus, the cyclophosphamide dosage was effective in preclinical models of medulloblastoma when administered in combination, although it was not associated with a clinically relevant systemic exposure for cyclophosphamide or its metabolites (data not shown).

The plasma-ECF pharmacokinetic model that was developed for cyclophosphamide and its metabolites during this study will be of interest to analyze future pharmacodynamic data (i.e., tumor growth inhibition profile) in preclinical mouse models of medulloblastoma, and establish exposureresponse relationships. However, it should be acknowledged that the current model can not be directly translated for use in pediatric patients. For simplicity and identifiability issues, our model didn't account for metabolic processes occurring in the brain and/or the tumor. It was assumed that the extent of metabolism occurring in the brain was negligible compared to that occurring in the liver. Thus, only the transfer plasma-to-ECF and ECF-to-plasma rates were modeled, with the primary goal of characterizing the ECF exposures of cyclophosphamide and its two metabolites. The inter-species differences found in terms of metabolism and protein binding as well as differences in the transporters' expression and activity, and physiological fluid flows that govern CNS penetration will have to be accounted by using more physiological CNS pharmacokinetic models that also include brain tissue and cerebrospinal fluid compartments, as these are the data that were reported for cyclophosphamide in humans instead of ECF concentrations $(36,37)$. Further work will be required as it may be challenged by the complex metabolic profile of cyclophosphamide involving multiple enzymatic systems which may differ between species.

To summarize, this study provided new and additional information into the extent to which cyclophosphamide, 4OH-CTX, and CEPM cross the blood-brain barrier in non-tumor-bearing mice and in mice bearing G3MB tumors. These results will be used in the context of future efficacy analyses evaluating combination treatments in mouse models of medulloblastoma to characterize 
cyclophosphamide exposure-response relationships, and to guide the development of new therapies.

\section{ACKNOWLEDGMENTS}

We thank Dana Farmer for growing and providing G3MB tumor cells for implantation and the Veterinary Pathology Core. This work was supported by grants from the National Cancer Institute (CA21765 (MFR; CFS), CA096832 (MFR), and R25CA23944), and the American Lebanese Syrian Associated Charities (ALSAC) at St. Jude Children's Research Hospital.

\section{REFERENCES}

1. Khanna V, Achey RL, Ostrom QT, Block-Beach H, Kruchko C, Barnholtz-Sloan JS, et al. Incidence and survival trends for medulloblastomas in the United States from 2001 to 2013. J Neurooncol. 2017;135(3):433-41.

2. Northcott PA, Korshunov A, Witt H, Hielscher T, Eberhart CG, Mack S, et al. Medulloblastoma comprises four distinct molecular variants. J Clin Oncol. 2011;29(11):1408-14.

3. Taylor MD, Northcott PA, Korshunov A, Remke M, Cho YJ, Clifford SC, et al. Molecular subgroups of medulloblastoma: the current consensus. Acta Neuropathol. 2012;123(4):465-72.

4. Cavalli FMG, Remke M, Rampasek L, Peacock J, Shih DJH, Luu B, et al. Intertumoral Heterogeneity within Medulloblastoma Subgroups. Cancer Cell. 2017;31(6):737-54 e6.

5. Gajjar A, Bowers DC, Karajannis MA, Leary S, Witt H, Gottardo NG. Pediatric Brain Tumors: Innovative Genomic Information Is Transforming the Diagnostic and Clinical Landscape. J Clin Oncol. 2015;33(27):2986-98.

6. Friedman HS, Bigner SH, Bigner DD. Cyclophosphamide therapy of medulloblastoma: from the laboratory to the clinic and back again (and again and again). J Neurooncol. 1995;24(1):103-8.

7. Allen JC, Helson L. High-dose cyclophosphamide chemotherapy for recurrent CNS tumors in children. J Neurosurg. 1981;55(5):749-56.

8. Gajjar A, Kuhl J, Epelman S, Bailey C, Allen J. Chemotherapy of medulloblastoma. Childs Nervous System. 1999;15(10):554-62.

9. White L, Sterling-Levis K. Multiagent chemotherapy studied in a xenograft model of medulloblastoma/primitive neuroectodermal tumour: analysis of the VETOPEC regimen. Journal of clinical neuroscience : official journal of the Neurosurgical Society of Australasia. 2008;15(1):4954.
10. Morfouace M, Shelat A, Jacus M, Freeman BB, 3rd, Turner D, Robinson S, et al. Pemetrexed and gemcitabine as combination therapy for the treatment of Group3 medulloblastoma. Cancer Cell. 2014;25(4):516-29.

11. Endersby R, Whitehouse J, Hii H, Greenall SA, Johns TG, Gottardo NG. A Pre-Clinical Assessment of the Pan-ERBB Inhibitor Dacomitinib in Pediatric and Adult Brain Tumors. Neoplasia. 2018;20(5):432-42.

12. Genka S, Deutsch J, Stahle PL, Shetty UH, John V, Robinson $\mathrm{C}$, et al. Brain and plasma pharmacokinetics and anticancer activities of cyclophosphamide and phosphoramide mustard in the rat. Cancer Chemother Pharmacol. 1990;27(1):17.

13. Talha MR, Rogers HJ, Trounce JR. Distribution and pharmacokinetics of cyclophosphamide in the rat. $\mathrm{Br}$ J Cancer. 1980;41(1):140-3.

14. Arndt CA, Balis FM, McCully CL, Colvin OM, Poplack DG. Cerebrospinal fluid penetration of active metabolites of cyclophosphamide and ifosfamide in rhesus monkeys. Cancer Res. 1988;48(8):2113-5.

15. de Jonge ME, Huitema AD, Rodenhuis S, Beijnen JH. Clinical pharmacokinetics of cyclophosphamide. Clin Pharmacokinet. 2005;44(11):1135-64.

16. Kalhorn TF, Ren S, Howald WN, Lawrence RF, Slattery JT. Analysis of cyclophosphamide and five metabolites from human plasma using liquid chromatography-mass spectrometry and gas chromatography-nitrogen-phosphorus detection. J ChromatogrB BiomedSciAppl. 1999;732(2):287-98.

17. Kalhorn TF, Howald WN, Cole S, Phillips B, Wang J, Slattery JT, et al. Rapid quantitation of cyclophosphamide metabolites in plasma by liquid chromatography-mass spectrometry. J Chromatogr B Analyt Technol Biomed Life Sci. 2006;835(1-2):10513.

18. Jacus MO, Throm SL, Turner DC, Patel YT, Freeman $\mathrm{BB}, 3 \mathrm{rd}$, Morfouace $\mathrm{M}$, et al. Deriving therapies for children with primary CNS tumors using pharmacokinetic modeling and simulation of cerebral microdialysis data. Eur J Pharm Sci. 2014;57:41-7.

19. de Lange EC, Danhof M, de Boer AG, Breimer DD. Methodological considerations of intracerebral microdialysis in pharmacokinetic studies on drug transport across the blood-brain barrier. Brain research Brain research reviews. 1997;25(1):27-49.

20. Beal SL. Ways to fit a PK model with some data below the quantification limit. J Pharmacokinet Pharmacodyn. 2001;28(5):481-504.

21. Nguyen TH, Mouksassi MS, Holford N, Al-Huniti N, Freedman I, Hooker AC, et al. Model Evaluation of Continuous Data Pharmacometric Models: Metrics and Graphics. CPT: pharmacometrics \& systems pharmacology. 2017;6(2):87-109. 
22. Holford NH. The Visual Predictive Check Superiority to Standard Diagnostic (Rorschach) Plots. PAGE 14, Abstr 738 [http://www.pagemeeting.org/?abstract=972] 2005

23. D'Argenio DZ. Optimal sampling times for pharmacokinetic experiments. J Pharmacokinet Biopharm. 1981;9(6):739-56.

24. D'Argenio DZ, Schumitzky A, Wang X. ADAPT 5 user's guide: pharmacokinetic/pharmacodynamic systems analysis software. Los Angeles: Biomedical Simulations Resource; 2009.

25. Di L, Rong H, Feng B. Demystifying brain penetration in central nervous system drug discovery. Miniperspective. J Med Chem. 2013;56(1):2-12.

26. Daryani VM, Patel YT, Tagen M, Turner DC, Carcaboso AM, Atkinson JM, et al. Translational Pharmacokinetic-Pharmacodynamic Modeling and Simulation: Optimizing 5-Fluorouracil Dosing in Children With Pediatric Ependymoma. CPT: pharmacometrics \& systems pharmacology. 2016;5(4):211-21.

27. Vogelbaum MA. Targeted Therapies for Brain Tumors: Will They Ever Deliver? Clin Cancer Res. 2018;24(16):3790-1.

28. McCune JS, Salinger DH, Vicini P, Oglesby C, Blough DK, Park JR. Population pharmacokinetics of cyclophosphamide and metabolites in children with neuroblastoma: a report from the Children's Oncology Group. J Clin Pharmacol. 2009;49(1):88102.

29. Navid F, Baker SD, McCarville MB, Stewart CF, Billups CA, Wu J, et al. Phase I and Clinical Pharmacology Study of Bevacizumab, Sorafenib, and Low-Dose Cyclophosphamide in Children and Young Adults with Refractory/Recurrent Solid Tumors. Clinical Cancer Research. 2013;19(1):23646.

30. Qiu R, Yao A, Vicini P, McDonald GB, Batchelder $\mathrm{AL}$, Bouvier ME, et al. Diminishing the risk of nonrelapse mortality in hematopoietic stem cell transplantation: Prediction of exposure to the cyclophosphamide metabolite carboxyethylphosphoramide mustard. Clin Pharmacol Ther. 2004;76(3):270-80.

31. Martignoni M, Groothuis GM, de Kanter R. Species differences between mouse, rat, dog, monkey and human CYP-mediated drug metabolism, inhibition and induction. Expert opinion on drug metabolism \& toxicology. 2006;2(6):875-94.

32. Bagley CM, Jr., Bostick FW, DeVita VT, Jr. Clinical pharmacology of cyclophosphamide. Cancer Res. 1973;33(2):226-33.

33. Zhang J, Tian Q, Yung Chan S, Chuen Li S, Zhou S, Duan W, et al. Metabolism and transport of oxazaphosphorines and the clinical implications. Drug Metab Rev. 2005;37(4):611-703.

34. Morfouace M, Cheepala S, Jackson S, Fukuda Y, Patel YT, Fatima S, et al. ABCG2 Transporter Expression Impacts Group 3 Medulloblastoma Response to Chemotherapy. Cancer Research. 2015;75(18):3879-89.

35. Friedman HS, Colvin OM, Kaufmann SH, Ludeman SM, Bullock N, Bigner DD, et al. Cyclophosphamide resistance in medulloblastoma. Cancer Res. 1992;52(19):5373-8.

36. Yamamoto Y, Valitalo PA, Huntjens DR, Proost JH, Vermeulen A, Krauwinkel W, et al. Predicting Drug Concentration-Time Profiles in Multiple CNS Compartments Using a Comprehensive Physiologically-Based Pharmacokinetic Model. CPT: pharmacometrics \& systems pharmacology. 2017;6(11):765-77.

37. Yamamoto Y, Valitalo PA, Wong YC, Huntjens DR, Proost JH, Vermeulen A, et al. Prediction of human CNS pharmacokinetics using a physiologically-based pharmacokinetic modeling approach. Eur J Pharm Sci. 2018;112:168-79. 


\section{SUPPLEMENTAL MATERIAL}

\section{Section 1: Bioanalytical methods}

For quantitation of cyclophosphamide/carboxyethylphosphoramide mustard (CEPM) in mouse plasma, $25 \mu \mathrm{L}$ plasma samples were used for solid phase extraction. $75 \mu \mathrm{L}$ of $50 \mathrm{mM}$ ammonium acetate $(\mathrm{pH}=3)$ was added to plasma samples to adjust the $\mathrm{pH}$, followed by addition of $4 \mu \mathrm{L}$ of combined ISTD working solution at 12500 $\mathrm{ng} / \mathrm{mL}$ for both cyclophosphamide- $d_{4}$ and CEPM- $d_{8}$. The spiked plasma samples were loaded onto the Oasis HLB $\mu$ Elution plate which was pre-conditioned with $100 \mu \mathrm{L}$ methanol and then washed with $200 \mu \mathrm{LDDH}_{2} \mathrm{O}$. The plate was washed by $100 \mu \mathrm{L}$ of $5 \%$ methanol and then eluted with $100 \mu \mathrm{L}$ of $50 \%$ methanol in $\mathrm{DDH}_{2} \mathrm{O} .10 \mu \mathrm{L}$ sample extract was injected for LC-MS/MS analysis.

For quantitation of 4-hydroxycyclophosphamiode (4OH-CTX) in mouse plasma, $25 \mu \mathrm{L}$ plasma sample was transferred to $1.4 \mathrm{~mL}$ V-bottom pushcap tubes and $4 \mu \mathrm{L}$ of $500 \mathrm{ng} / \mathrm{mL}$ CEPM- $d_{8}$ working solution added followed by protein precipitation with $100 \mu \mathrm{L}$ methanol. The sample was vortexed for $1 \mathrm{~min}$ and centrifuged at $2680 \mathrm{xg}$ for $5 \mathrm{~min}$ at $4^{\circ} \mathrm{C} .80-100 \mu \mathrm{L}$ of the supernatant was transferred to autosampler vial and $12 \mu \mathrm{L}$ was injected for LCMS/MS analysis.

For quantitation of cyclophosphamide/CEPM and 4OH-CTX in Ringer's/derivatizing solution (2:1, v/v), 25 $\mu \mathrm{L}$ Ringer's/derivatizing solution samples was spiked with $4 \mu \mathrm{L}$ of combined ISTD working solution at 12500 $\mathrm{ng} / \mathrm{ml}$ for both cyclophosphamide- $d_{4}$ and CEPM- $d_{8}$, followed by dilution with $30 \mu \mathrm{L}$ methanol. $6 \mu \mathrm{L}$ sample was injected for LCMS/MS analysis.

LC/MS/MS analysis was performed using Shimadzu HPLC coupled with AB Sciex Qtrap 4000 mass spectrometry. Positive electrospray ionization (ESI) was used. For analysis of cyclophosphamide/CEPM in mouse plasma extracts, chromatography separation was achieved using Gemini C18 column $(3 \mu \mathrm{m}, 50 \mathrm{~mm} \times 4.6 \mathrm{~mm})$ and gradient elution with $0.1 \%$ formic acid in $\mathrm{H}_{2} \mathrm{O}$ as mobile phase $\mathrm{A}$ and methanol as mobile phase $\mathrm{B}$. The gradient program was set as $0-2 \mathrm{~min}, 60-85 \% \mathrm{~B}$; $2-2.5 \mathrm{~min}, 85-85 \% \mathrm{~B}$; $2.5-2.51 \mathrm{~min}, 85-60 \% \mathrm{~B}$; $2.51-6 \mathrm{~min}, 60$ $60 \% \mathrm{~B}$. Ringer's/derivatizing samples were analyzed using the same column and $0.1 \%$ formic acid in $\mathrm{H}_{2} \mathrm{O}$ as mobile phase $\mathrm{A}$ and $0.1 \%$ formic acid in methanol as mobile phase B. To achieve better separation, an extended gradient elution was used as $0-2 \mathrm{~min}, 10-10 \% \mathrm{~B}$; $2-5 \mathrm{~min}, 10-85 \% \mathrm{~B}$; $5-6.5 \mathrm{~min}, 85-85 \% \mathrm{~B}$; $6.5-7 \mathrm{~min}, 85-10 \% \mathrm{~B}$; 7-8 $\mathrm{min}, 10-10 \% \mathrm{~B}$. For analysis of $4 \mathrm{OH}-\mathrm{CTX}$ in mouse plasma extracts and Ringer's/derivatizing samples, chromatography separation was achieved using Gemini C18 column $(3 \mu \mathrm{m}, 100 \mathrm{~mm}$ x $4.6 \mathrm{~mm})$ and gradient elution with $0.1 \%$ formic acid in water as mobile phase $\mathrm{A}$ and methanol as mobile phase $\mathrm{B}$. The gradient program was set as $0-4.0 \mathrm{~min}, 55-95 \% \mathrm{~B} ; 4.0-4.50 \mathrm{~min}, 95-95 \% \mathrm{~B} ; 4.51-5.50 \mathrm{~min}, 55-55 \% \mathrm{~B}$. For both mouse plasma and Ringer's/derivatizing cyclophosphamide/CEPM gradient methods, the flow rate was set as $0.7 \mathrm{~mL} / \mathrm{min}$; while for $4 \mathrm{OH}-\mathrm{CTX}$ gradient methods, the flow rate was $1.0 \mathrm{~mL} / \mathrm{min}$. The column temperature was set at $30^{\circ} \mathrm{C}$ for the mouse plasma and Ringer's/derivatizing cyclophosphamide/CEPM and 4OH-CTX methods. Specific analytes and their corresponding internal standards were identified by retention time using the following precursor-to-product ion transitions, determined using analytical standards: $\mathrm{m} / \mathrm{z} 261 \rightarrow 140$ (cyclophosphamide), m/z $293 \rightarrow 221$ $(\mathrm{CEPM}), \mathrm{m} / \mathrm{z} 367 \rightarrow 221(4 \mathrm{OH}-\mathrm{CTX}) \mathrm{m} / \mathrm{z} 265 \rightarrow 235.2$ (cyclophosphamide- $d_{4}$ ), and $\mathrm{m} / \mathrm{z} 301 \rightarrow 229\left(\mathrm{CEPM}-d_{8}\right)$. CEPM- $\mathrm{d}_{8}$ was used as internal standard for both CEPM and 4OH-CTX analyses. The calibration range for cyclophosphamide, CEPM and 4OH-CTX in mouse plasma was $10-10000 \mathrm{ng} / \mathrm{mL}, 60-6000 \mathrm{ng} / \mathrm{mL}$ and $4.73-$ $9074.07 \mathrm{ng} / \mathrm{mL}$ respectively; while that in Ringer's/derivatizing solution was 5-2000 ng/mL, 20-1000 ng/mL and $1.89-4253.57 \mathrm{ng} / \mathrm{mL}$ for cyclophosphamide, CEPM and 4OH-CTX, respectively. 
Figure

S1.
Cyclophosphamide

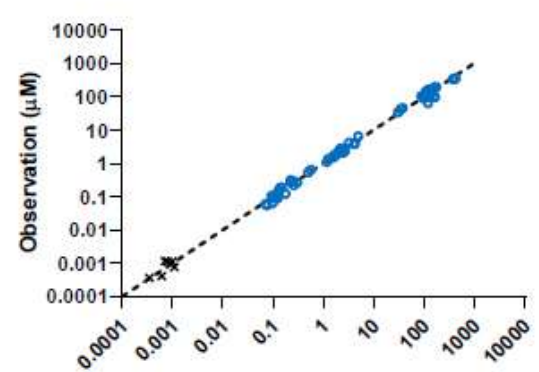

Individual predictions $(\mu \mathrm{M})$
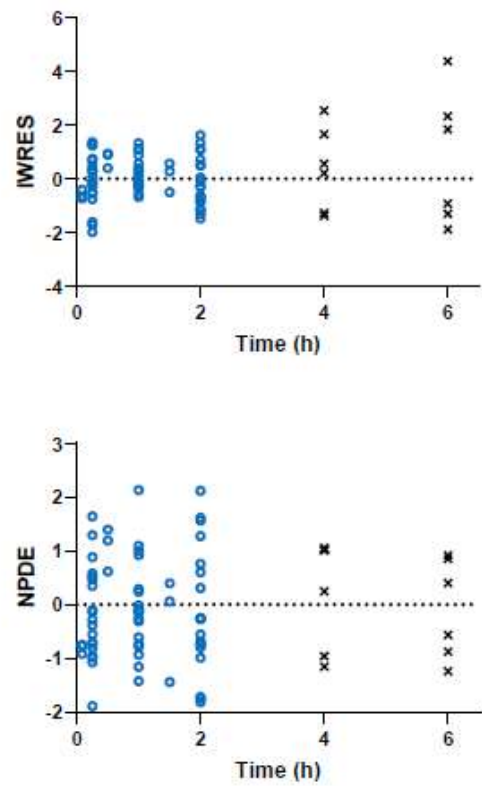

4OH-CTX
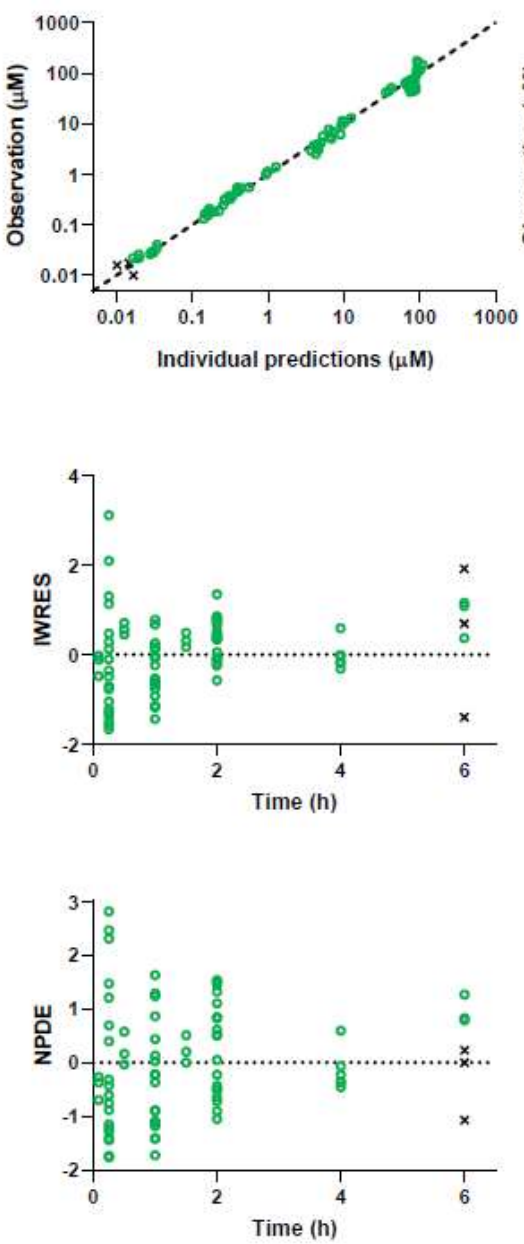

CEPM
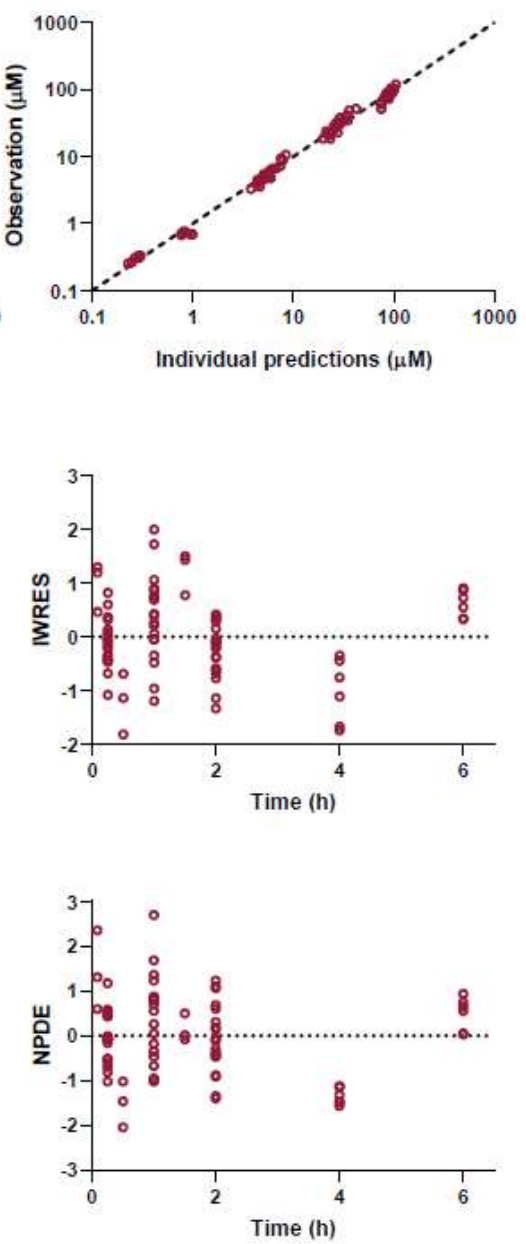

Goodness-of-fit plots for the plasma pharmacokinetic model.

The first, second, and third columns represent the diagnostic plots for cyclophosphamide, 4-hydroxy-cyclophosphamide (4OH-CTX), and carboxyethylphosphoramide mustard (CEPM), respectively. The first row depicts the observed plasma concentrations $v s$ the individual model predictions. The dashed lines are the unity lines. The second row depicts the individual weighted residuals vs time, and the third row shows the normalized predicted distribution errors $v s$ time. In these graphs, the dashed lines represent the $\mathrm{y}=0$ line. Crosses represent data below the limit of quantification.

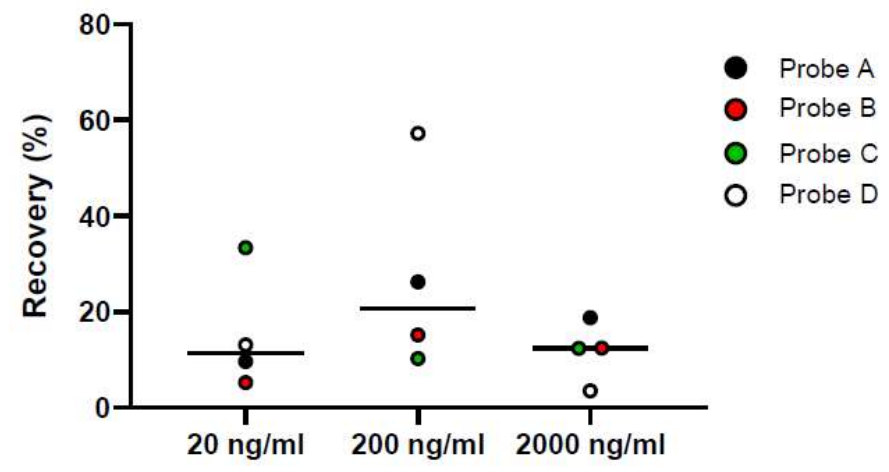

Cyclophosphamide concentration

Figure S2. In vitro recovery coefficients obtained for each tested probe at different cyclophosphamide concentrations. 


\section{Cyclophosphamide}

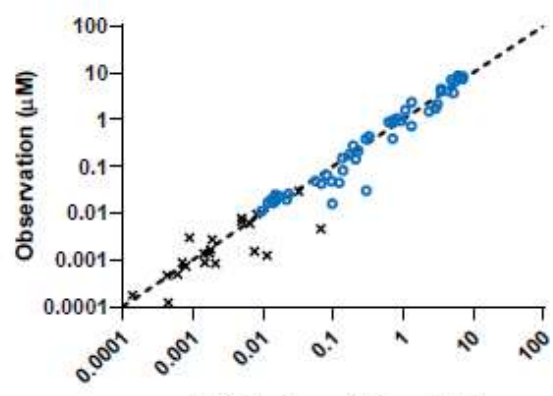

Individual predictions $(\mu \mathrm{M})$
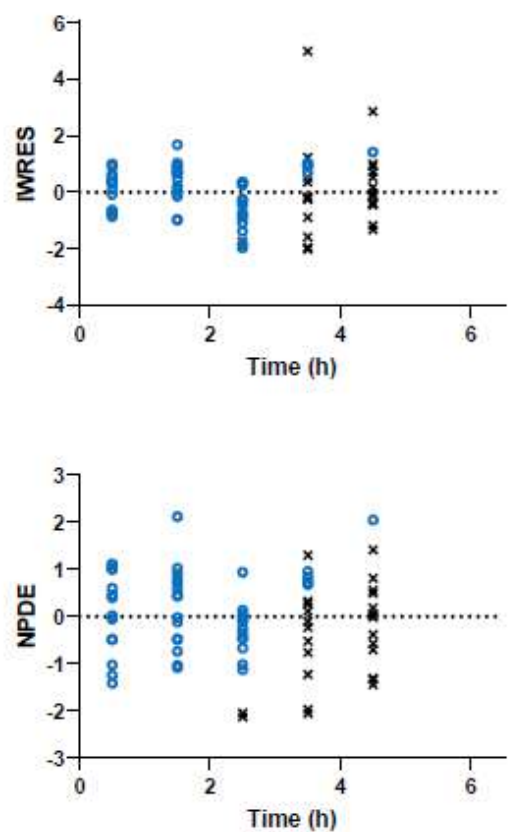

4OH-CTX
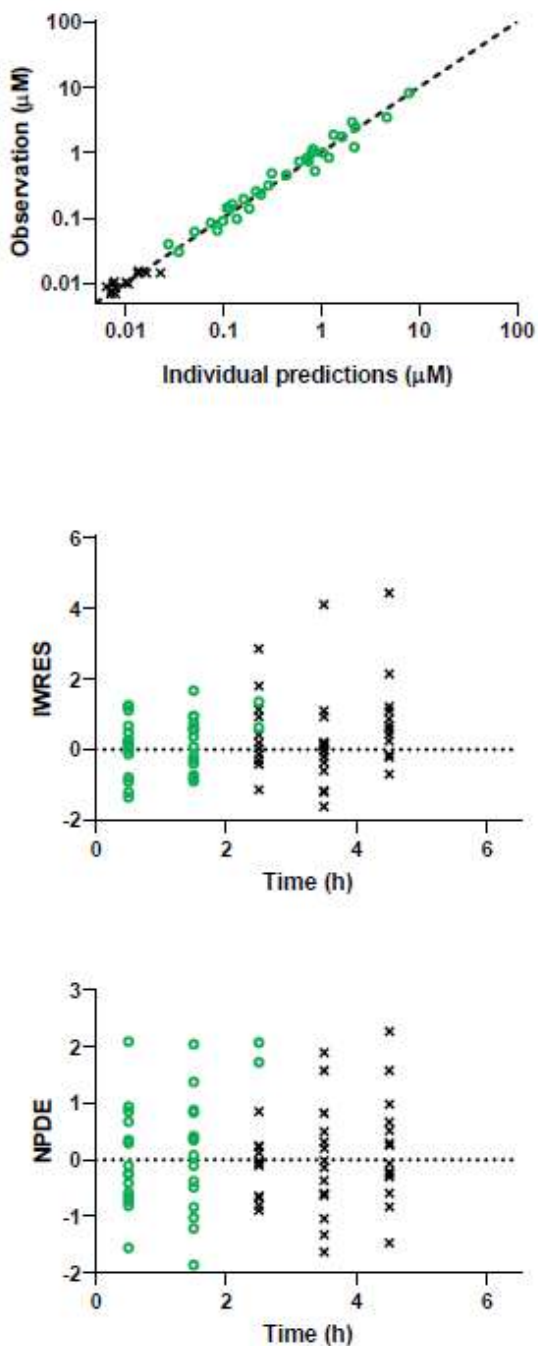

CEPM
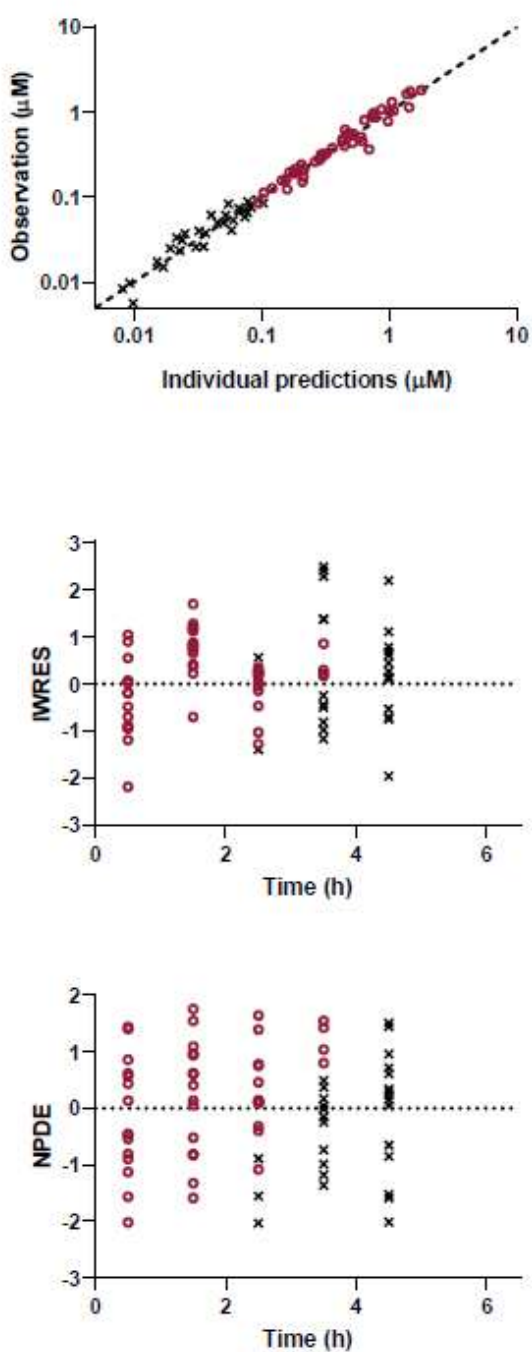

Figure S3. Goodness-of-fit plots for the ECF pharmacokinetic model.

The first, second, and third columns represent the diagnostic plots for cyclophosphamide, 4-hydroxy-cyclophosphamide (4OH-CTX), and carboxyethylphosphoramide mustard (CEPM), respectively. The first row depicts the observed ECF concentrations $v s$ the individual model predictions. The dashed lines are the unity lines. The second row depicts the individual weighted residuals $v s$ time, and the third row shows the normalized predicted distribution errors $v s$ time. In these graphs, the dashed lines represent the $\mathrm{y}=0$ line. Crosses represent data below the limit of quantification. 


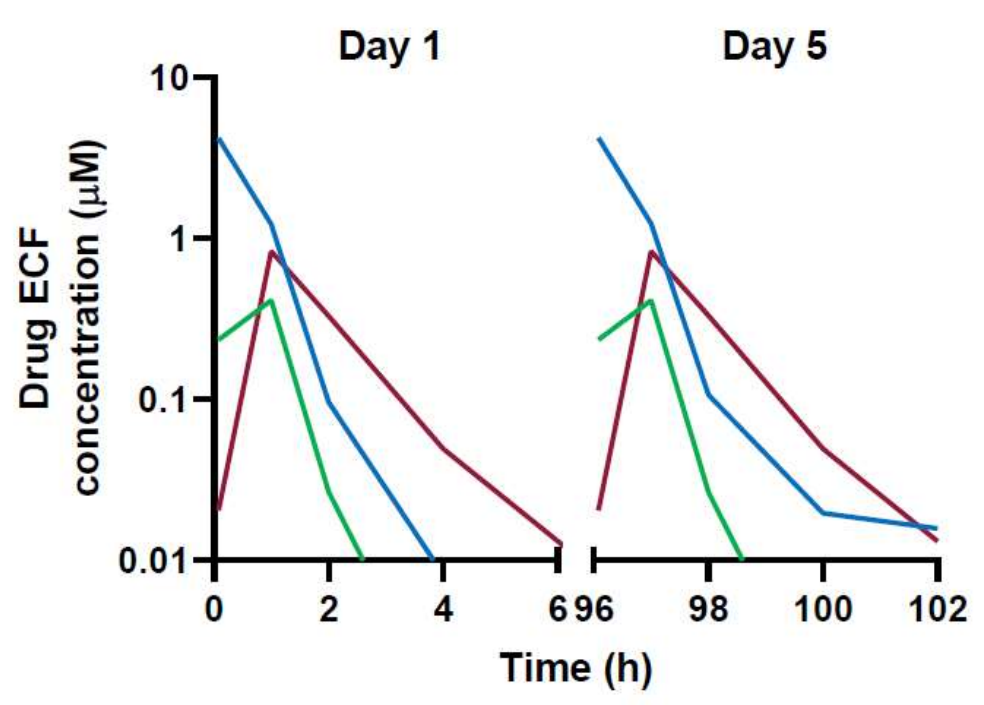

Figure S4. Mean model-simulated ECF concentration-time profiles for cyclophosphamide (blue), 4OH-CTX (green) and CEPM (red) after a dosage regimen of $130 \mathrm{mg} / \mathrm{kg}$ cyclophosphamide IP daily for five consecutive days. 